\title{
REVIEW
}

Open Access

\section{Efficacy assessment of mesenchymal stem cell transplantation for burn wounds in animals: a systematic review}

\author{
Hanxiao $\mathrm{Yi}^{1 \dagger}$, Yang Wang ${ }^{2 \dagger}$, Zhen Yang ${ }^{1 *}$ and Zhiqin Xie ${ }^{1}$
}

\begin{abstract}
Background: Clinically, severe burns remain one of the most challenging issues, but an ideal treatment is yet absent. Our purpose is to compare the efficacy of stem cell therapy in a preclinical model of burn wound healing.

Methods: Research reports on mesenchymal stem cells (MSCs) for burn wound healing were retrieved from 5 databases: PubMed, Embase, MEDLINE, Web of Science, and the Cochrane Library. The primary outcomes reported in this article include the un-healing rate of the wound area, the closure rate, and the wound area. Secondary outcomes included CD-31, vascular density, interleukin (IL)-10, thickness of eschar tissue, vascular endothelial growth factor (VEGF), and white blood cell count. Finally, a subgroup analysis was conducted to explore heterogeneity that potentially impacted the primary outcomes. A fixed-effects model with a 95\% confidence interval (Cl) was performed when no significant heterogeneity existed. Otherwise, a random-effects model was used. All data analysis was conducted by using Engauge Digitizer 10.8 and R software.
\end{abstract}

Results: Twenty eligible articles were finally included in the analysis. Stem cell therapy greatly improved the closure rate $(2.00,95 \% \mathrm{Cl} 0.52$ to $3.48, p=0.008)$ and compromised the wound area $(-2.36 ; 95 \% \mathrm{Cl}-4.90$ to $0.18 ; p=0.069)$ rather than the un-healing rate of the wound area $(-11.10,95 \% \mathrm{Cl}-32.97$ to $10.78, p=0.320)$. Though $p$ was 0.069 , there was a trend toward shrinkage of the burn wound area after stem cell therapy. Vascular density (4.69; $95 \% \mathrm{Cl} 0.06$ to $9.31 ; p=0.047)$ and thickness of eschar tissue $(6.56,95 \% \mathrm{Cl} 1.15$ to $11.98, p=0.017)$ were also discovered to be significantly improved in the burn site of stem cell-treated animals. Moreover, we observed that animals in the stem cell group had an increased white blood cell count $(0.84,95 \% \mathrm{Cl} 0.01$ to $1.66, p=$ $0.047) 5$ days post treatment. Other indicators, such as VEGF $(p=0.381), \operatorname{CD}-31 \quad(p=0.335)$ and IL-10 ( $p=0.567)$, were not significantly impacted.

Conclusions: Despite limited data from preclinical trials, this meta-analysis suggests that stem cell therapy is curative in decreasing the burn wound area and provides some insights into future clinical studies of stem cell therapy for burns.

Keywords: Burn wound, Stem cell therapy, Animals, Efficacy, Meta-analysis

\footnotetext{
* Correspondence: yangzhen11@hotmail.com

${ }^{\dagger}$ Hanxiao Yi and Yang Wang contributed equally to this work.

${ }^{1}$ The First Affiliated Hospital of Nanchang University, No. 17, Yongwai

Zhengjie, Nanchang, JiangXi Province, China

Full list of author information is available at the end of the article
}

\section{$\triangle B M C$}

(c) The Author(s). 2020 Open Access This article is licensed under a Creative Commons Attribution 4.0 International License, which permits use, sharing, adaptation, distribution and reproduction in any medium or format, as long as you give appropriate credit to the original author(s) and the source, provide a link to the Creative Commons licence, and indicate if changes were made. The images or other third party material in this article are included in the article's Creative Commons licence, unless indicated otherwise in a credit line to the material. If material is not included in the article's Creative Commons licence and your intended use is not permitted by statutory regulation or exceeds the permitted use, you will need to obtain permission directly from the copyright holder. To view a copy of this licence, visit http://creativecommons.org/licenses/by/4.0/ The Creative Commons Public Domain Dedication waiver (http://creativecommons.org/publicdomain/zero/1.0/) applies to the data made available in this article, unless otherwise stated in a credit line to the data. 


\section{Introduction}

According to the World Health Organization (WHO), an estimated 265,000 people worldwide annually die from burns, and even nonfatal burns are a major cause of illness [1,2]. Burns are classified into three degrees: first degree (the superficial layer of the epidermis), second degree (dermis), and third degree (full-thickness skin, even reaching the muscle and bone). Burns are the consequence of chemicals [3], irradiation [4], heat [5], and electricity [6] and potentially lead to dehydration, electrolyte imbalance, infections, and even death. Severe burns are a devastating injury that affects almost all organs and causes severe morbidity and mortality [7], and no ideal treatments for severe burns have been recommended.

Existing clinical management protocols include improved resuscitation and treatment of inhalation injuries (for patients with respiratory injuries), early debridement and closure of wounds, appropriate infection control, and metabolic support [2, 8, 9]. However, significant challenges remain. At the onset of the loss of skin integrity, this injury can result in fragile protective competence of the first physical barrier, blood loss, severe pain, and subsequent infections, especially during the process of healing, which is both time and nutrient consuming. For patients with extensive burns and limited donor skin, skin grafts are sometimes reticulated and expanded to achieve greater coverage. However, mesh grafts may take several weeks to mature, and the cosmetic results are often unsatisfactory to patients. In addition, complications such as infections or skin contracture may occur, leading to additional treatments and a prolonged healing process $[10,11]$. Therefore, new treatments are urgently needed.

Currently, mesenchymal stem cells (MSCs) are widely reported to involve many pathophysiological processes [12-14] and have a curative effect in treating various diseases [15-17]. MSCs, which can differentiate into epidermal cells and skin appendages (sebaceous 1and sweat glands), can be isolated and amplified from the umbilical cord, bone marrow, fat, and other tissues and have recently been reported to decrease burn the wound area in rats and mice [14, 18]. Despite the multipotency of stem cells, an increasing number of studies have shown that the efficacy of MSCs primarily depends on the secretion of a variety of factors, such as vascular endothelial growth factor (VEGF), epidermal growth factor (EGF), fibroblast growth factor (FGF), insulin-like growth factor (IGF), plateletderived factor growth factor (PDGF), transforming growth factor beta (TGF beta), IL-4, IL-6, and IL-10, which play an important role in angiogenesis, cell recruitment, immune regulation, and wound healing, rather than the stemness of cells [19].
Currently, a series of clinical trials (1 allogenic MSC, 1 cadaveric MSC, 1 bone marrow MSC) and a casecontrol prospective study (bone marrow $\mathrm{MSC}=20$, umbilical cord-MSC $=20, E E \& G=20$ ) investigating the efficacy and safety of stem cell therapy have been completed. The results demonstrate that MSCs can effectively promote burn wound healing and are considered safe and effective, with great therapeutic potential for patients with severe burns [20-23]. However, to date, there has been no systematic and comprehensive preclinical analysis of stem cell therapy for burn wounds, and the integration of these preclinical data can offset knowledge gaps that may affect the future application of stem cells. Therefore, we conducted a systematic review and meta-analysis of animal studies of the use of MSCs for treating burn wounds.

\section{Methods and materials Search strategy}

The items of this meta-analysis were reported according to the Preferred Reporting Items for Systematic Review and Meta-Analysis (PRISMA) [24] (see Additional file 1).

We searched the PubMed, Embase, MEDLINE, Web of Science, and Cochrane Library databases systematically and comprehensively (from onset to April 2020). To obtain as many articles as possible, the search strategy "((mesenchymal stem cells) OR MSCs) AND burn" was used in our search, and the species was set as "other animals" rather than "human beings" (see Additional file 2). In addition, other studies that may have been eligible were manually identified by references or other reviews related to this topic.

\section{Selection criteria}

The primary inclusion criteria were the use of stem cells through the administration of exogenous stem cells to promote post-hyperthermia wound healing and skin regeneration after burn injury. Stem cells are referred to as pluripotent and multipotent progenitor cells with the ability to self-renew and differentiate into organ- or tissue-specific cells. In summary, studies employing stem cells from bone marrow, umbilical cord, and fat and reporting any aspects of burn wounds were included in the analysis.

The exclusion criteria were the use of embryonic stem cells (ESCs), progenitor cells (epithelial and dermal stem cells), and programmed stem cells. Nonthermal burns (such as chemical and electricity burns), injury to organs and mucosa other than skin (such as the esophagus and respiratory tract), and old burns were excluded. Studies that reported unexpected outcomes and outcomes of humans were eliminated. Studies that used self-control were excluded as well. 


\section{Data extraction}

From the included studies, we extracted the following data: (a) identity: author and year of publication, design; (b) animals for each study: number of animals, species, age, burn model, region, stem cell type; (c) treatment: dosage, administration, timing (post burn). Animal experiments differ from randomized clinical trials (RCTs) and retrospective studies from humans, as there are no suitable assessment strategies. Thus, a quality assessment of each study was not conducted.

The data used for analysis were independently analyzed by two authors (Yi Hanxiao and Wang Yang). If a contradiction occurred, the original article was rechecked to confirm the accuracy of the data.

\section{Types of outcome measures}

The following indicators were used to evaluate the efficacy of MSCs in treating burn wounds: closure rate and un-healing rate of the wound area, and wound area. The closure rate and un-healing rate of the wound area were expressed as percentages of the area, while the wound area was the actual area $\left(\mathrm{mm}^{2}\right)$ reported in the original articles and this meta-analysis. CD-31 and vascular density were used to assess the status of angiogenesis by immunohistochemical staining, expressed as the average optical density (AOD) and number of vascular rings, respectively. VEGF and IL-10 were detected by reverse transcription-polymerase chain reaction (RT-QPCR) and enzyme-linked immunosorbent assay (ELISA), respectively. Other indicators, such as white blood cell count and eschar tissue thickness, were also evaluated.

\section{Statistical analysis}

This meta-analysis was conducted using $\mathrm{R}$ software 3.63 (University of Auckland, New Zealand). All data evaluated in this article were continuous data and are expressed as the standardized mean difference (SMD) with corresponding 95\% CI to eliminate the influence from units and measures. The chi-squared value test and inconsistency index $\left(I^{2}\right)$ were used to assess the heterogeneity across each study. A random-effects model was used to analyze the data if a value of $p<0.05$ or $I^{2}>$ $50 \%$, which was considered to have significant heterogeneity. Otherwise, a fixed-effects model was used. Subgroup analysis was used to find potential sources of heterogeneity. A leave-one-out meta-analysis and metaregression were conducted to explore potential heterogeneities. Publication bias was tested by Egger's test $t$ with $\mathrm{R}$ software (University of Auckland, New Zealand)

\section{Results}

\section{Study screen}

According to our search strategy, 683 articles were finally retrieved from PubMed, MEDLINE, Cochrane
Library, Web of Science, and Embase databases. After elimination of duplicates, 664 articles were retained. Articles were further screened by browsing their titles and abstracts; 620 articles were excluded as irrelevant to the topic, and a total of 44 articles were determined for fulltext review. Following careful review, 22 articles were removed as a result of the absence of anticipated outcomes associated with burn wounds. Eventually, 20 studies with parallel groups were included in this meta-analysis (Fig. 1).

\section{Study characteristics}

The baseline characteristics of all studies are shown in Table 1. Published studies from 2003 to 2019, with 5 studies from China, 5 studies from the USA, 2 studies from Iran, 2 studies from Taiwan, and 1 each from Russian, Ireland, Turkey, Japan, and New Orleans, and Pakistan. The sample size ranged from 3 to 74 . Most of the studies were conducted on rats and mice (18 out of 20), 2 of which were conducted on pigs. The studied cell types included bone MSCs (BMSCs, $n=6$ ), adiposederived MSCs (ADMSCs, $n=10$ ), and umbilical cord MSCs (UC-MSCs, $n=4$ ). The cell dosage ranging from $10^{4}$ to $10^{6}$ cells/wound was used immediately or 9 days post treatment. In 16 studies (80\%), local injection and tail injection were performed, and in 3 studies (15\%), stem cell-treated sterile dressings were applied. Other items, such as the objective of the study, species, age, and burn model, were also included.

\section{Primary results Closure rate}

Our primary goal was to assess whether stem cells had a therapeutic effect on burn wound healing, while the primary outcome was composed of three aspects: closure rate, un-healing rate of the wound area, and wound area. The closure rate and un-healing rate of the wound area were expressed as percentages of the area, and the wound area was expressed as the actual area of the burned site.

Closure rates were reported by 8 studies. We divided the outcome of closure rate into 4 groups: 7 days $(n=6)$, 10 days $(n=1), 14$ days $(n=7)$, and 21 days $(n=3)$. In total, 256 animals and 312 burn wounds were included in the analysis (Fig. 2). Only Mahmood [10] reported the closure rate on the 10th day; thus, the result from Mahmood was not pooled. On the 7th day, animals in the MSC group already had a higher closure rate $(0.61,95 \%$ CI 0.11 to $1.12, p=0.002$ ) than animals in the control group. On the 14th day, the outcome shared similar significance $(2.00,95 \%$ CI 0.52 to $3.48, p=0.008)$ with the outcome on the 7th day. However, no significant difference $(-1.96,95 \% \mathrm{CI}-6.82$ to $2.90, p=0.428)$ in the 


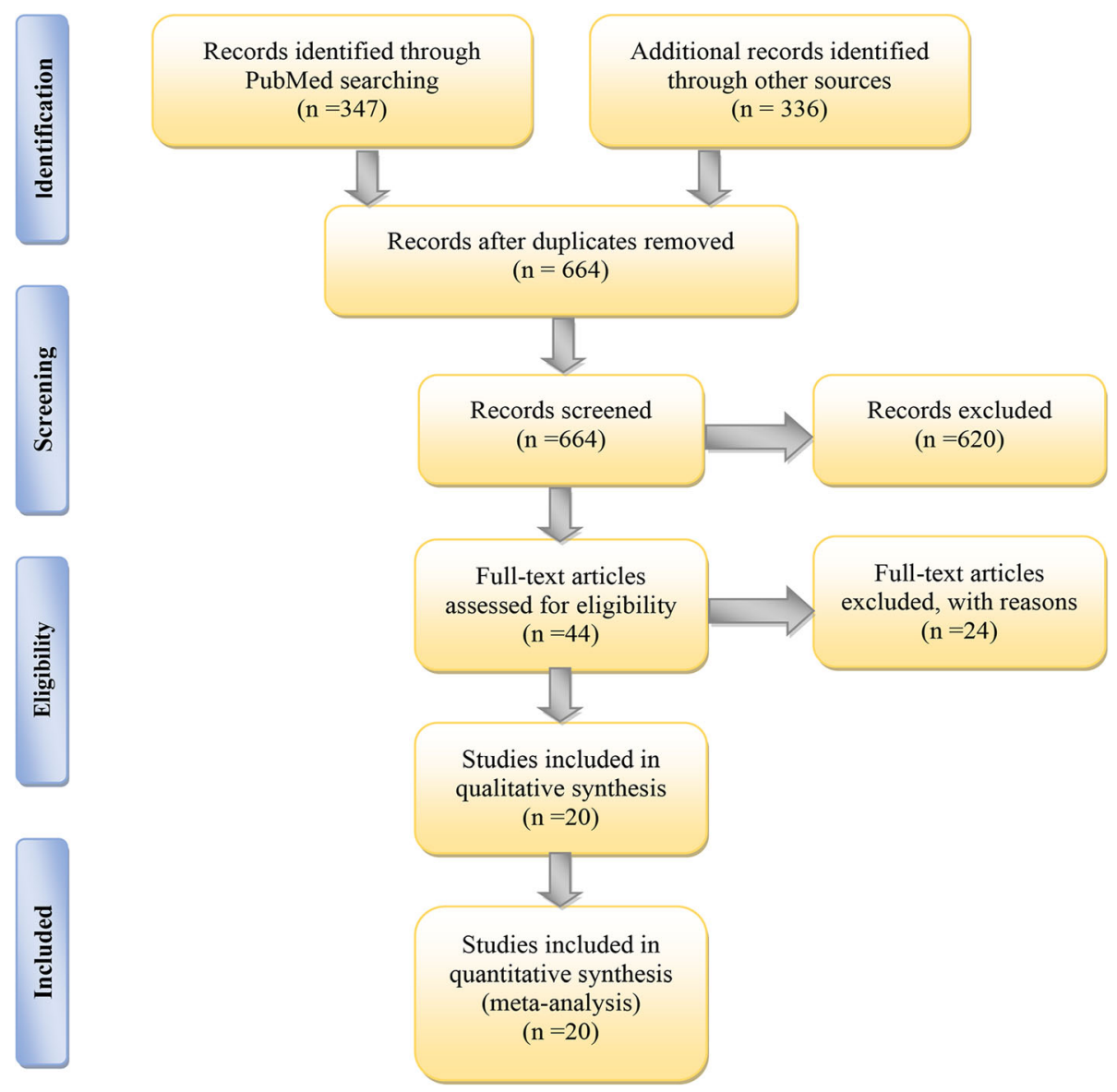

Fig. 1 Flowchart of the article screening process

closure rate was observed between the two groups on the 21 st day.

A leave-one-out meta-analysis was also conducted to determine that elimination of articles by Kaita [25] changed the outcome of the closure rate to nonsignificance (0.56, 95\% CI -0.04 to 1.16$)$ on the 7 th day (see Additional file 3). On the 14th day, no article was observed to greatly impact the final outcome of closure rate (see Additional file 4).

\section{Wound area}

Four studies, including 62 animals and 80 burn wounds, reported the outcome of wound area at the 1st and 2nd week. This indicator was no longer reported by Shumakov [26] but was reported by three other authors at the 3rd week. Similarly, we observed that wound area was negatively associated with stem cell therapy at the 1st ($1.80,95 \% \mathrm{CI}-3.74$ to $0.15, p=0.070)$, 2nd $(-1.29,95 \%$ CI -2.81 to $0.24, p=0.098)$, and 3rd weeks $(-2.36,95 \%$ CI -4.90 to $0.18, p=0.069$ ). Although nonsignificance was discovered at each time point, we observed that the $p$ value from each group approached 0.05 , which suggested a closing trend of the wound area (Fig. 3).
A leave-one-out meta-analysis revealed that the wound area greatly decreased if articles by Karimi $(-3.30,95 \%$ CI -6.40 to -0.19$)$ or Bliley $(-3.40$, 955 CI -6.61 to 0.19 ) were omitted at the 1st week (see Additional file 5). However, at the 2nd and 3rd weeks, no article that significantly impacted the final outcome was observed (see Additional files 6 and 7).

\section{Un-healing rate of wound area}

The un-healing rate of the wound area was only reported by 2 articles, including 23 animals and 112 burn wounds. Animals in the experimental group had the same un-healing rate of the wound area as the animals in the control group (95\% CI -32.97 to $10.78, p=0.320)$ (Fig. 4).

\section{Secondary results}

In this section, we primarily focused on biochemical events (such as the white blood cell count, eschar thickness, CD-31, vascular density, IL-10, and VEGF) that occurred following stem cell administration (Table 2). A total of 5 comparative studies involving 127 animals and 178 burn wounds evaluated the effect of MSCs on the 


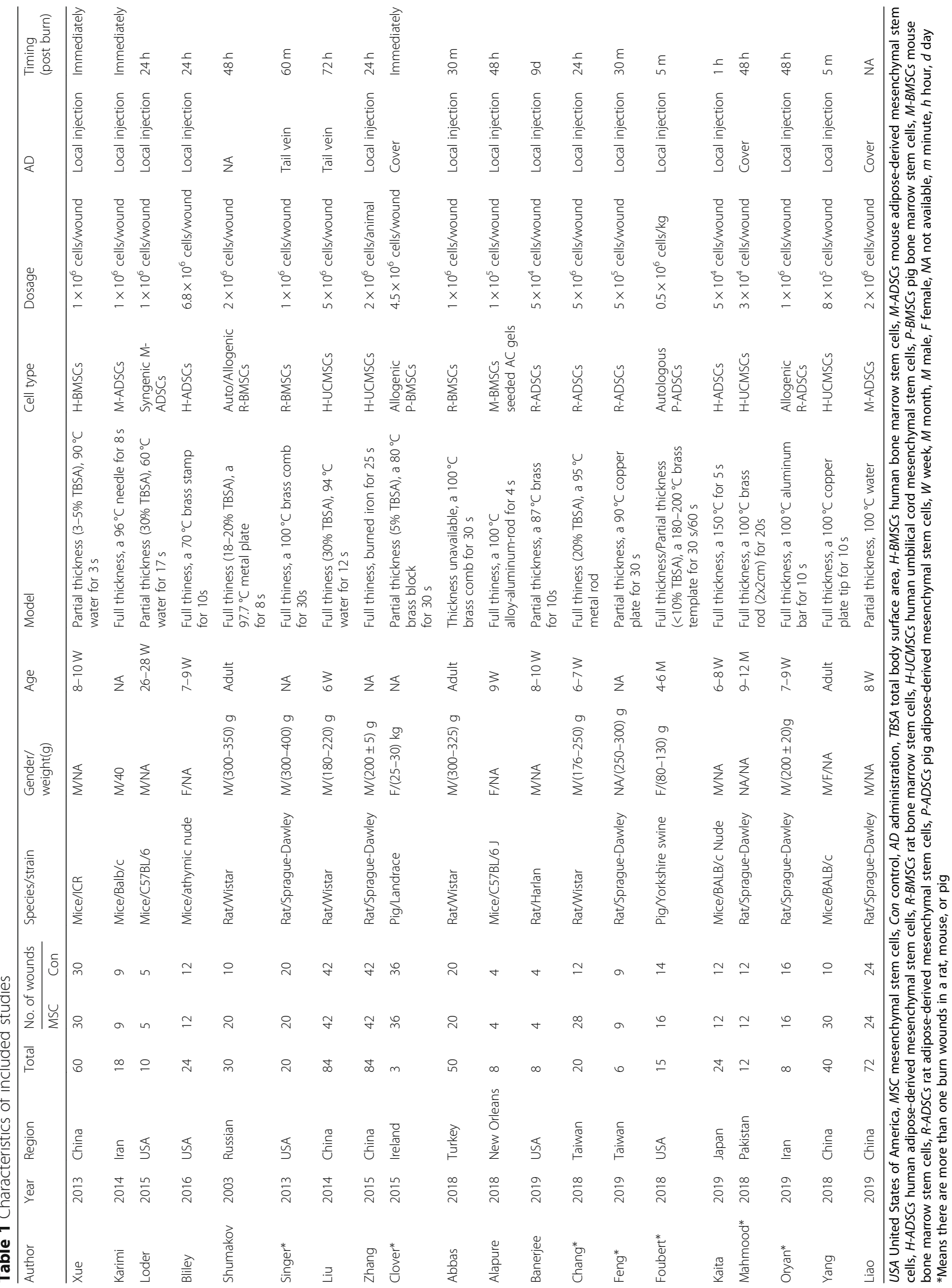




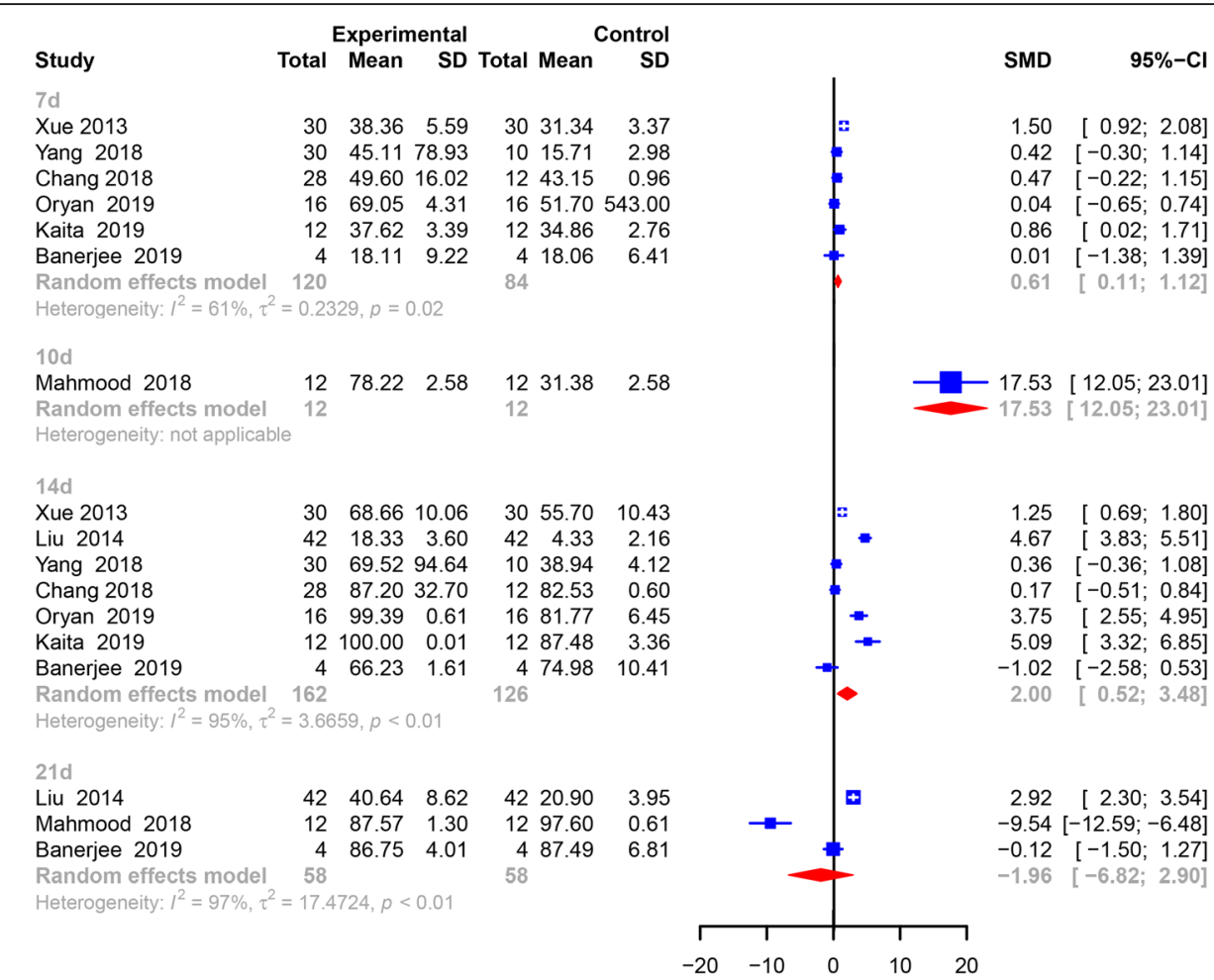

Fig. 2 Primary outcome of closure rate. The outcome of the closure rate was analyzed at four different time points. All analyses showed positive results for the shrinking closure rate $(p<0.05)$. All analyses were conducted by using a random-effects model with a $95 \%$ confidence interval

thickness of the eschar tissue and the vascular density. The pooled analysis showed that experimental animals had a thicker scar $(6.56,95 \%$ CI 1.115 to $11.98, p=$ $0.017)$ and a higher vascular density $(4.69,95 \%$ CI 0.06 to 9.31, $p=0.047)$ ) than animals administered no treatment and animals treated with phosphate buffer solution
(PBS). Interestingly, we observed a negative result in CD-31 staining $(10.02,95 \% \mathrm{CI}-10.33$ to $30.37, p=$ 0.335 ), which was contradictory to the pooled analysis of vascular density.

Both Zhang and Foubert [27, 28] continuously reported changes in the white blood cell count of 27

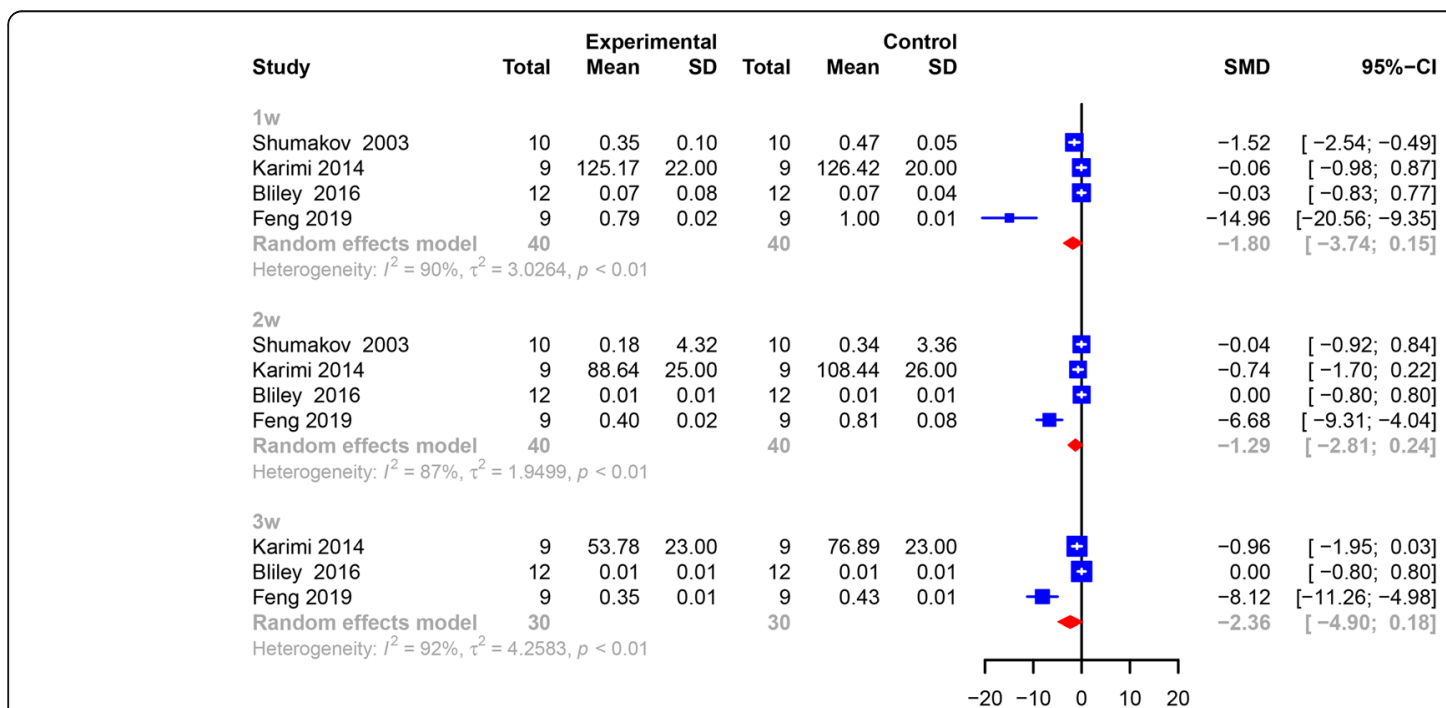

Fig. 3 Primary outcome of the wound area. The outcome of closure rate was analyzed every other week, and a negative association was observed between MSC administration and wound area. All analyses were conducted by using a random-effects model with a $95 \%$ confidence interval 


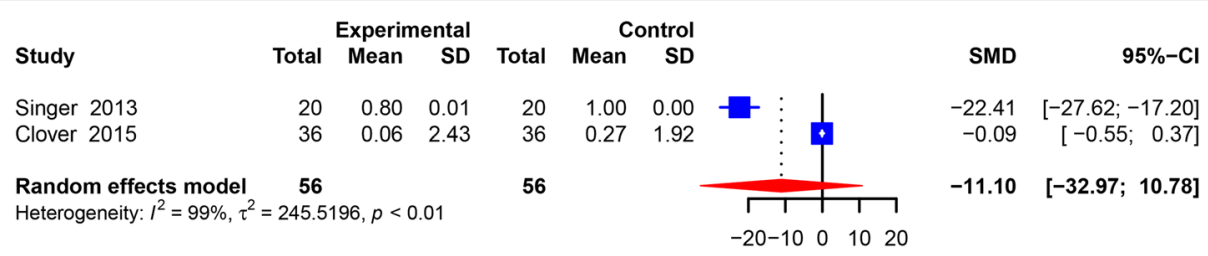

Fig. 4 Primary outcome of the un-healing rate of the wound area. The pooled analysis suggested that both groups shared a similar closure rate of un-healing of the wound area. The analysis was conducted by using a random-effects model with a $95 \%$ confidence interval

animals at $0,1,2,3$, and 5 days post treatment (Fig. 5). At the $0(0.32,95 \% \mathrm{CI}-0.44$ to $1.09, p=0.409)$, 1 st $(0.02,95 \%$ CI -0.73 to $0.78, p=0.953)$, 2nd $(-3.39$, $95 \%$ CI -11.71 to $4.93, p=0.424)$, and 3rd day $(-1.16$, $95 \% \mathrm{CI}-4.81$ to $2.49, p=0.533$ ) after treatment, no significant change in the white blood cell count was discovered in the MSC group until the 5th day post treatment. On the last day, an elevated count of white blood cells $(0.84,95 \%$ CI 0.01 to $1.66, p=0.047)$ was observed in animals administered MSCs.

Other factors, such as IL-10 $(9.82,95 \% \mathrm{CI}-23.78$ to $43.41, p=0.567)$ and VEGF $(-2.95,95 \% \mathrm{CI}-9.55$ to $3.65, p=0.381)$, were not significantly increased in animals in the MSC group.

\section{Subgroup analysis}

To identify heterogeneity potentially influencing the analysis of the healing process, articles were divided into several groups on the basis of year, species, cell type, cell origin, gender, and region. On the 7 th day, pooled analysis of articles within 5 years showed a 0.39 higher closure rate $(95 \%$ CI 0.03 to $0.74, p=0.000)$ in the MSC group. MSC therapy seemed to significantly elevate the closure rate for mice regardless of MSC type (1.03, 95\% CI 0.63 to $1.43, p=0.000)$ and animals treated with MSCs of human origin $(0.65,95 \%$ CI 0.04 to $1.26, p=$ $0.038)$. Stem cells from rats did not show a curative effect on the closure rate $(0.23,95 \% \mathrm{CI}-0.23$ to $0.69, p=$ $0.325)$. In articles published by Asian authors (0.67, $95 \%$ CI 0.13 to $1.21, p=0.015)$ and in studies involving male animals $(0.65,95 \%$ CI 0.04 to $1.26, p=0.038)$, there was also a significant difference in closure rate between the two groups (Table 3) at 14 days post treatment (DPT).

Table 2 Secondary outcomes

\begin{tabular}{llll}
\hline Item & SMD & $95 \% \mathrm{Cl}$ & $P$ value \\
\hline Thickness of eschar & 6.56 & {$[1.15 ; 11.98]$} & $\mathbf{0 . 0 1 7}$ \\
CD-31 & 10.02 & {$[-10.33 ; 30.37]$} & 0.335 \\
Vascular density & 4.69 & {$[0.06 ; 9.31]$} & $\mathbf{0 . 0 4 7}$ \\
IL-10 & 9.82 & {$[-23.78 ; 43.41]$} & 0.567 \\
VEGF & -2.95 & {$[-9.55 ; 3.65]$} & 0.381 \\
\hline
\end{tabular}

$S M D$ standardized mean difference, IL interleukin, $C D$-31 cluster of differentiation-31, VEGF vascular endothelial growth factor
Even though a pooled analysis resulted in no significant differences between the two groups regardless of whether the articles were from within 5 years $(p=0.070)$ or beyond 5 years $(p=0.085)$, a trend of significance was discovered. Moreover, for mice treated with all sorts of MSCs $(3.33,95 \%$ CI 0.01 to $6.65, p=0.049)$ and animals treated with MSCs of human origin (3.33, 95\% CI 0.01 to $6.65, p=0.049$ ), a significant difference in the closure rate between the two groups was identified. On the 14th day, Asian articles also showed a great improvement in closure rate $(2.46,95 \%$ CI $0.89,4.02, p=0.002)$ (Table 4).

For the wound area, subgroup analysis of species, cell origin, and cell type at the 1st, 2nd, and 3rd week was conducted. Regardless of the period post treatment, no factor of species, cell origin, and cell type had a significant impact on the final outcome of the wound area. However, the wound area tended to decrease in animals treated with adipose-derived stem cells at the 1 st $(-2.42$, 95\% CI -5.22 to $0.38, p=0.091)$ and 2 nd $(-2.01,95 \%$ CI -4.34 to $0.32, p=0.091)$ weeks (Table 5 ).

\section{Publication bias}

Publication bias was tested by funnel plots (Fig. 6) and Egger's linear regression in closure rate and wound area individually. As the number of included studies that measured the closure rate and wound area was small $(<$ 10), it remained unclear whether the scattered points were the result of publication bias or the limited number of articles.

No significant publication bias was observed for the closure rate on the 7th (Egger's test, $p=0.329$ ) or 14th (Egger's test, $p=0.4316$ ) days. Although significant publication bias was discovered for wound area at the 3rd week (Egger's test, $p=0.060$ ) post treatment, significant publication bias was observed for wound area at the 1st week (Egger's test, $p=0.045$ ) post treatment, which may be the result of the small sample effect.

Furthermore, the outcomes of meta-regression demonstrated that species $(p=0.058)$, cell type $(p=0.005)$, cell origin $(p=0.058)$, and year $(p=0.001)$ were potential heterogeneous factors, rather than gender $(p=0.757)$ or region $(p=0.463)$, for closure rate on the 7 th day. However, on the 14th day, year $(p=0.708)$, gender $(p=$ 


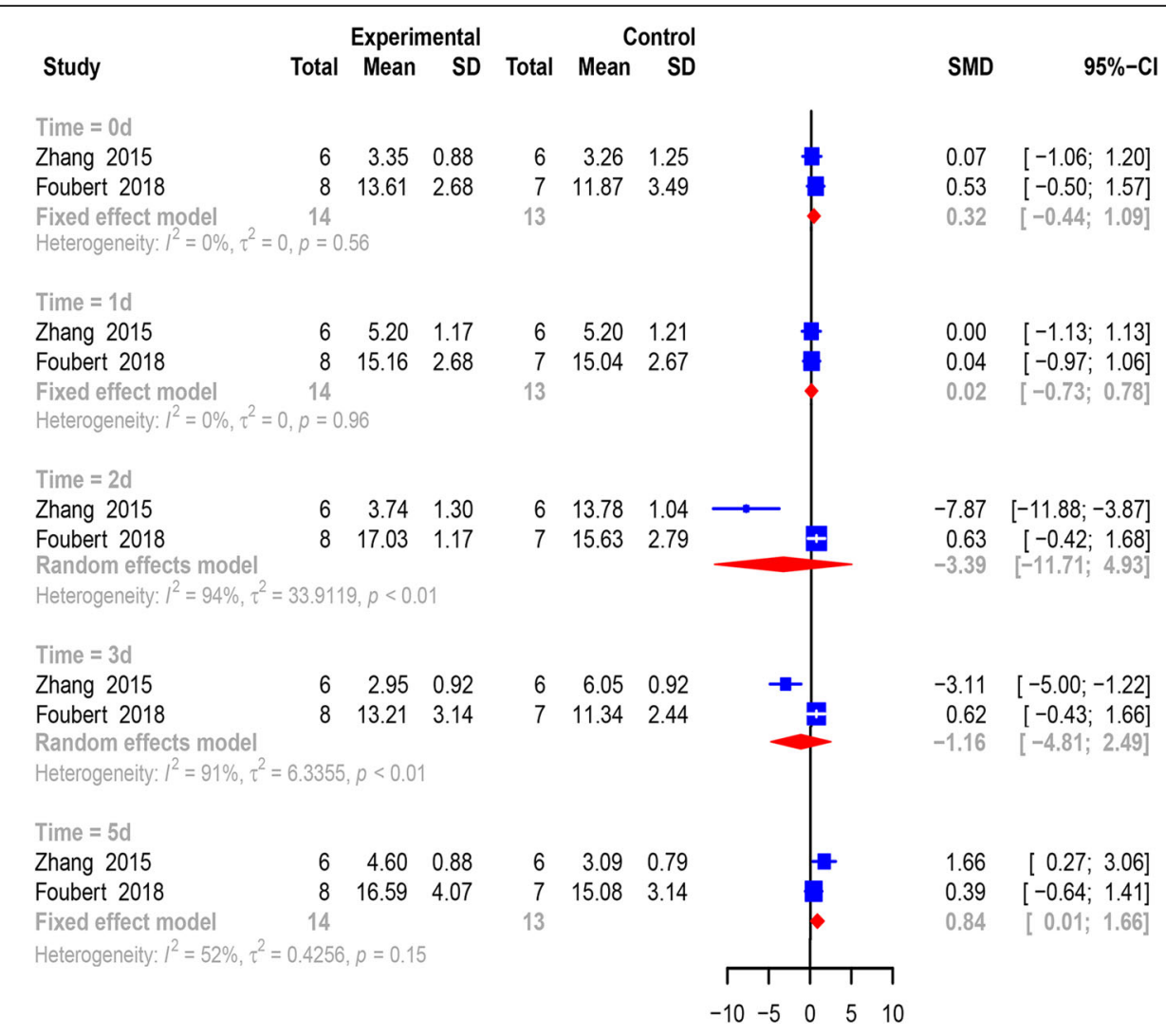

Fig. 5 Pooled analysis of the white blood cell count. Significant changes in white blood cells were not observed within 3 days. On the fifth day, white blood cells were greatly elevated in animals receiving MSC administration. All analyses were conducted by using a random- or fixed-effects model with a 95\% confidence interval

Table 3 Subgroup analysis for the closure rate at 7 DPT

\begin{tabular}{llll}
\hline Subgroup & SMD & $95 \% \mathrm{Cl}$ & $\boldsymbol{P}$ value \\
\hline Year & & & \\
Within 5 years & 0.39 & {$[0.03,0.74]$} & $\mathbf{0 . 0 3 2}$
\end{tabular}

\section{Species}

$\begin{array}{llll}\text { Mouse } & 1.03 & {[0.63,1.43]} & \mathbf{0 . 0 0 0} \\ \text { Rat } & 0.23 & {[-0.23,0.69]} & 0.325\end{array}$

\section{Cell type}

$\begin{array}{llll}\text { ADSCS } & 0.23 & {[-0.23,0.69]} & 0.325\end{array}$

\section{Cell origin}

$\begin{array}{llll}\text { Human } & 1.03 & {[0.63,1.43]} & \mathbf{0 . 0 0 0} \\ \text { Rat } & 0.23 & {[-0.23,0.69]} & 0.325 \\ \text { Gender } & & & \\ \text { Male } & 0.65 & {[0.04,1.26]} & \mathbf{0 . 0 3 8}\end{array}$

Region

$\begin{array}{llll}\text { Asia } & 0.67 & {[0.13,1.21]} & \mathbf{0 . 0 1 5}\end{array}$

DPT days post treatment, SMD standardized mean difference

Table 4 Subgroup analysis for the closure rate at 14 DPT

\begin{tabular}{|c|c|c|c|}
\hline Subgroup & SMD & $95 \% \mathrm{Cl}$ & $\boldsymbol{P}$ value \\
\hline \multicolumn{4}{|l|}{ Year } \\
\hline Beyond 5 years & 2.95 & {$[-0.41,6.03]$} & 0.085 \\
\hline Within 5 years & 1.59 & {$[-0.13,3.32]$} & 0.070 \\
\hline \multicolumn{4}{|l|}{ Species } \\
\hline Mouse & 3.33 & {$[0.01,6.65]$} & 0.049 \\
\hline Rat & 1.07 & {$[-0.39,2.52]$} & 0.151 \\
\hline \multicolumn{4}{|l|}{ Cell type } \\
\hline ADSCs & 1.07 & {$[-0.39,2.52]$} & 0.151 \\
\hline \multicolumn{4}{|l|}{ Cell origin } \\
\hline Human & 3.33 & {$[0.01,6.65]$} & 0.049 \\
\hline Rat & 1.07 & {$[-0.39,2.52]$} & 0.151 \\
\hline \multicolumn{4}{|l|}{ Gender } \\
\hline Male & 2.29 & {$[0.55,4.03]$} & 0.010 \\
\hline \multicolumn{4}{|l|}{ Region } \\
\hline Asia & 2.46 & {$[0.89,4.02]$} & 0.002 \\
\hline
\end{tabular}


Table 5 Subgroup analysis for the wound area at 1st, 2nd, and 3 rd weeks post treatment

\begin{tabular}{|c|c|c|c|}
\hline Subgroup & SMD & $95 \% \mathrm{Cl}$ & $\boldsymbol{P}$ value \\
\hline \multicolumn{4}{|l|}{$1 \mathrm{st}$} \\
\hline \multicolumn{4}{|l|}{ Species } \\
\hline Mouse & -0.04 & {$[-0.65,0.56]$} & 0.8895 \\
\hline Rat & -7.94 & {$[-21.10,5.22]$} & 0.2369 \\
\hline \multicolumn{4}{|c|}{ Cell origin } \\
\hline Mouse & -7.24 & {$[-21.83,7.35]$} & 0.3309 \\
\hline \multicolumn{4}{|l|}{ Cell type } \\
\hline ADSCs & -2.42 & {$[-5.22,0.38]$} & 0.0908 \\
\hline \multicolumn{4}{|l|}{ 2nd } \\
\hline \multicolumn{4}{|l|}{ Species } \\
\hline Mouse & -0.32 & {$[-1.04,0.40]$} & 0.3837 \\
\hline Rat & -3.24 & {$[-9.74,3.26]$} & 0.3289 \\
\hline \multicolumn{4}{|l|}{ Cell origin } \\
\hline Mouse & -3.58 & {$[-9.39,2.24]$} & 0.2279 \\
\hline \multicolumn{4}{|l|}{ Cell type } \\
\hline ADSCs & -2.01 & {$[-4.34,0.32]$} & 0.0912 \\
\hline \multicolumn{4}{|l|}{$3 r d$} \\
\hline \multicolumn{4}{|l|}{ Species } \\
\hline Mouse & -0.43 & {$[-1.37,0.50]$} & 0.3639 \\
\hline \multicolumn{4}{|c|}{ Cell origin } \\
\hline Mouse & -4.38 & {$[-11.39,2.63]$} & 0.2212 \\
\hline
\end{tabular}

SMD standardized mean difference

$0.402)$, species $(p=0.163)$, cell type $(p=0.185)$, cell origin $(p=0.163)$, and region $(p=0.114)$ were not potential heterogeneous origins. For wound area, both cell origin $(p=0.7324)$ and cell type $(p=0.8212)$ were not potential heterogeneous factors, in contrast with species $(p=$ $0.028)$, at the 1st week. However, cell origin $(p=0.692)$, cell type $(p=0.390)$, and species $(p=0.236)$ were not heterogeneous factors for wound area at the 2nd week. At the 3rd week, the meta-regression analysis suggested that species $(p=0.00)$, rather than cell origin $(p=0.473)$, was the source of potential heterogeneity.

\section{Discussion}

When combined with a meta-analysis of these experiments, a systematic review enables a more systematic and objective assessment of the results. Severe burns are one of the most devastating injuries [29, 30]. Burns are one of the most common traumas worldwide, with more than 300,000 people dying each year from fire-related burns and millions suffering from burn-induced deformities and mortality [31].

Stem cells are self-renewing cells that can differentiate into specific cell types. Pluripotent stem cells, i.e., ESC or induced pluripotent stem cells (iPSCs), differentiate into all three embryonic lines of cells. Since the 1970s, MSCs have been called mesenchymal stromal cells, and numerous achievements have been made in the field of regenerative therapy using these cells [32]. MSCs have some important advantages over other types of stem cells. MSCs are clongenic and able to differentiate into various cell lineages, including muscle, cartilage, fat, and bone [33], and they are multipotent, releasing a wide range of biologically active paracrine factors. Furthermore, MSCs have documented immunomodulatory properties and can be isolated with relative ease from a variety of adult tissues, such as bone marrow, tendon, adipose tissue, skin, and periodontal ligament [34-39]. Importantly, this flexibility in where MSCs can be harvested avoids ethical issues associated with the use of stem cells from embryonic cells. Finally, the potential risk of stem cell therapy is their ability to become malignant, which is especially true for iPSCs. In contrast, this unique risk appears to be reduced in MSC therapy. Previous studies have suggested that MSCs can significantly attenuate inflammation [40, 41] and tissue fibrosis, promote tissue remodeling despite the use of bleomycin treatment [42], and shorten the healing process with less scar formation in burn patients [43]. Over the years, the use of MSCs to accelerate burn wound closure has shown promising prospects [44-47]. The potential mechanisms associated with burn treatment could be attributed to abundant anti-inflammatory cytokines secreted by MSCs, such as VEGF, EGF, FGF, IGF, PDGF, TGF, IL-4, IL-6, and IL-10. Moreover, MSC treatment significantly reduces neutrophil infiltration and the levels of pro-inflammatory cytokines (TNF $\alpha, \mathrm{IL}-1 \beta$, and IL-6) while promoting anti-inflammatory cytokine IL-10 expression in the stagnation region [48]. Additionally, MSC-treated interstitial spaces exhibit increased expression of VEGF-A, PDGF, FGF, and TGF, increasing the vascular density in the lesion site [49].

Nevertheless, to the best of our knowledge, this is the first attempt to comprehensively collect and evaluate the current preclinical evidence supporting the use of MSCs in animal models of burn wounds. Our results demonstrated that MSCs are potentially therapeutic in burn animal models.

Overall, the results of our meta-analysis revealed an improvement in the wound closure rate and a trend toward shrinkage in the wound area. Pooled analysis of only two articles suggested a negative result in the unhealing rate of the wound area, which was an aspect that measured the degree of the burn injuries and led to a controversial conclusion. However, two articles may not firmly support this controversial conclusion, which was even inconsistent with the results reported by Motamed et al. [50] Sensitivity analysis showed a more stable outcome on the 14th day than the outcome of closure rate 

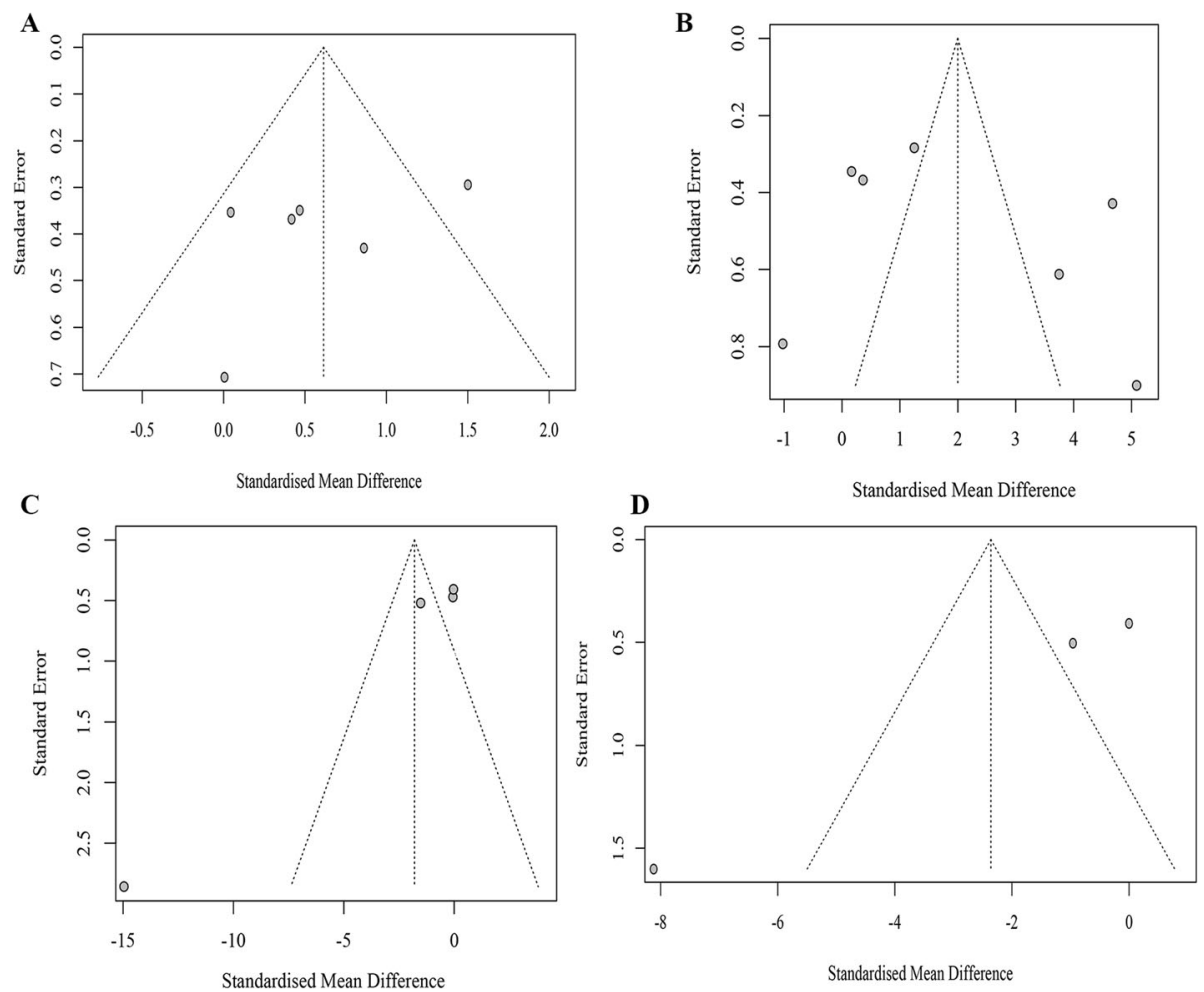

Fig. 6 Funnel plots of primary outcomes. Funnel plots were conducted for closure rate on the 7th (a) and 14th (b) days and for the wound area on the 1st (c) and 3rd (d) weeks

on the 7th day, as the omission of a study by Kaita [25] significantly impacted the result. We also evaluated the eschar thickness and vascular density in animals treated with regenerative cells, which were obviously better and consistent with the research results of Sultan et al. [51] Interestingly, animals receiving MSCs did not exhibit increased expression of CD-31, which may be explained by limited articles. Taken together, these findings support the potential use of MSC therapy in preclinical applications of burn wounds. To explore sources of heterogeneity, subgroup analyses and meta-regression were conducted on the basis of species, cell origin, cell type, gender, region, and year. The analysis revealed that these variables were exceedingly relevant for future patient application, species, cell type, and cell origin and were potential sources of heterogeneity; thus, the process of generalizing MSC therapy in humans may be conducted more cautiously. These findings provide a certain reference significance, which reminds us of some speciesand cell-related issues that are pertinent to future preclinical or clinical trials, and these variables need to be taken into account to determine the best successful outcome.

Despite the exclusion of other cell-based studies, we reviewed the efficacy of other cell-based therapies in the treatment of burn wounds. Shi [52] observed that the healing rate of the ESC group on days 7 and 14 was $26.0 \pm 2.0$ and $64.4 \pm 4.7 \%$, respectively, which was significantly higher than that of the control group (12.4 \pm 1.1 and $29.1 \pm 3.3 \%$ ). Khan [53] also observed that the treatment of wounds with iPSCs significantly promoted the rate of wound healing closure compared to that observed in other groups $(p<0.05)$. The mechanism by which HSCs accelerate the wound healing process potentially involves the secretion of collagen and downregulation of matrix metalloproteinase (MMP) expression [54]; meanwhile, endothelial progenitor cells (EPCs) participate in this process by promoting vessel density [55]. While all of the abovementioned cells can accelerate wound healing, the cell type that is the most suitable for burn wound healing remains unclear.

Despite positive findings in small animal models, the conversion to large animal models and clinical research has been limited. Of the three case human reports, no study described adverse effects. In a study by Jeschke et al [20], a male patient in his mid-20s with a $70 \%$ TBSA burn injury had wounds that remained unhealed after more than 18 months of routine burn care. With the administration of allogeneic MSCs, the open wound was reduced from one third to less than $3 \%$, and the infection was significantly cured in a short time. Furthermore, the wound sites showed no evidence of keloids or 
hypertrophic formation during a 6-year follow-up period. Another clinical trial by Wael et al. [23] also observed a significantly reduced hospitalization period in both the BM-MSCs and UC-MSCS groups as compared to the EE\&G group, indicating that mesenchymal stem cells of both bone marrow and cord blood origin can effectively improve the healing of burn injuries. Furthermore, all these studies described rapid improvement in overall clinical condition. However, the number of available studies is too small to enable a meta-analysis of the clinical application of MSC. One phase I clinical trial (NCT02104713) is currently underway in the USA to determine the safety of allogeneic stem cell therapy from healthy donors for 2 nd degree burn wounds of less than $20 \%$ TBSA at four different dose levels (ranging from $2.5 \times 10^{3}$ to $2 \times 10^{4}$ allogeneic BM-MSCs $/ \mathrm{cm}^{2}$ ) [56].

In addition to being used to treat burns, MSCs and other cells are also used to treat other traumatic and regenerative diseases alone or in combination with other agents (i.e., platelet-rich plasma (PRP) and hyaluronic acid). Hair follicle mesenchymal stem cells (HF-MSCs), human intra- and extradermal adipose tissue-derived hair follicle stem cells (HD-AFSCs), and stem cells with PDGF present safe and viable treatment alternatives against hair loss [57, 58]. In addition, PRP alone or in combination with hyaluronic acid can be used to regenerate lower-extremity complex wounds, treat severe hidradenitis suppurativa, and protect against hair loss [59-61]. Additionally, PRP is effective in the treatment of jaw regeneration, the protection of HUVECs, and promotion of the chondro/osteogenic differentiation of ADMSCs in the presence of insulin [62-64]. Gentile et al. observed that transplantation of nanofat was positively correlated with scar regeneration, and even the transplantation of Permacol, a porcine dermal matrix, was effective in treating an infected abdomen wound $[65,66]$. The regenerative effects of these cells could be attributed to paracrine through the secretion of various growth factors and the exocytosis of exosomes. Growth factors, such as EGF, FGF, and VEGF, strongly activate their receptor pathways to induce proliferation-associated pathways (Akt and Erk) and promote cell proliferation [67]. Furthermore, these cytokines (i.e., exosomes) can distinctly suppress inflammation cascades in the wound site, blocking the expansion of the secondary injury [68].

The advantages of this investigation are apparent. First, we are the first to conduct a meta-analysis of the impact of MSC therapy on burn wounds. Second, we conducted a systematic literature search and followed the published protocols to ensure a stringent review process. Third, the detailed analysis adds variables that the researcher needs to pay attention to.
Although our meta-analysis showed that MSC transplantation was effective in animal burn wound healing, some limitations should be taken into consideration. First, the results are from various species of animals rather than humans, which may lead to poor reproducibility from preclinical to clinical trials. Furthermore, some subgroups had a small sample size. Additionally, the overall number of studies is small. Additionally, we could not assess the quality of studies such as those investigating humans. Finally, we cannot evaluate the clinical safety of MSCs in treating burn wounds owing to the lack of reported side-effect events and the studied objectives. Finally, there was no in-depth study on the dose effect and administration method of MSCs on burn wounds.

\section{Conclusion}

Our pooled analysis showed that MSC therapy, which is an emerging treatment for burn wounds, is therapeutic in accelerating the process of wound healing in animals, and this study provides some insights into the clinical application of stem cell therapy. Owing to a lack of assessment of side-effect events for MSCs, safety issues should receive greater focus while clinical trials are ongoing.

\section{Supplementary information}

Supplementary information accompanies this paper at https://doi.org/10 1186/s13287-020-01879-1.

\footnotetext{
Additional file 1. PRISMA 2009 Checklist.

Additional file 2. Search Strategy.

Additional file 3. Leave-one-out meta-analysis of the closure rate on the 7th day. We conducted a sensitivity analysis by using a leave-one-out meta-analysis, showing that no single article (except for one study conducted by Kaita) significantly impacted the final pooled outcome of closure rate.
}

Additional file 4. Leave-one-out meta-analysis of the closure rate on the 14th day. The leave-one-out meta-analysis calculated with randomeffects models showed that the final closure rate was so stable that it could be significantly impacted by a single study.

Additional file 5. Leave-one-out meta-analysis of the wound area at the 1st week. The leave-one-out meta-analysis calculated with random-effects models showed that the final result of the wound area could be easily affected by two studies conducted by Karimi and Bliley.

Additional file 6. Leave-one-out meta-analysis of the wound area at the 2nd week. Leave-one-out meta-analysis calculated with random-effects models showed that the pooled outcome of the wound area was not significantly changed by a single article.

Additional file 7. Leave-one-out meta-analysis of the wound area at the 3rd week. Leave-one-out meta-analysis calculated with random-effects models showed that the pooled outcome of the wound area was not significantly changed by a single article.

\section{Abbreviations}

USA: United States of America; MSC: Mesenchymal stem cells; Con: Control; AD: Administration; TBSA: Total body surface area; H-BMSCs: Human bone marrow stem cells; M-ADSCs: Mouse adipose-derived mesenchymal stem cells; H-ADSCs: Human adipose-derived mesenchymal stem cells; R- 
BMSCs: Rat bone marrow stem cells; H-UCMSCs: Human umbilical cord mesenchymal stem cells; P-BMSCs: Pig bone marrow stem cells; MBMSCs: Mouse bone marrow stem cells; R-ADSCs: Rat adipose-derived mesenchymal stem cells; P-ADSCs: Pig adipose-derived mesenchymal stem cells; W: Week; M: Month; M: Male; F: Female; NA: Not available; m: Minute; h: Hour; d: Day; WBCs: White blood cells; SD: Standard difference; SMD: Standardized mean difference; IL: Interleukin; CD-31: Cluster of differentiation-31; VEGF: Vascular endothelial growth factor; DPT: Days post treatment; Cl: Confidence interval; WHO: World Health Organization; EGF: Epidermal growth factor; FGF: Fibroblast growth factor; IGF: Insulin-like growth factor; PDGF: Platelet-derived factor growth factor; TGF beta: Transforming growth factor beta; PRISMA: Preferred Reporting Items for Systematic Review and Meta-Analysis; ESCs: Embryonic stem cells; RCT: Randomized clinical trials; AOD: Average optical density; RTQPCR: Reverse transcription-polymerase chain reaction; ELISA: Enzyme-linked immunosorbent assay; PBS: Phosphate buffer solution; iPSCs: Induced pluripotent stem cells; MMP: Matrix metalloproteinase; EPCs: Endothelial progenitor cells; PRP: Platelet-rich plasma; HF-MSCs: Hair follicle mesenchymal stem cells; HD-AFSCs: Human intra- and extradermal adipose tissue-derived hair follicle stem cells

\section{Acknowledgements}

We are grateful for Yang Wang's assistance in extracting and analyzing the data.

\section{Authors' contributions}

ZY first presented the idea and designed the whole outline of this article. ZQ $X$ and $H X Y$ were responsible for all data extraction and analysis, with the assistance of YW. The final version was revised by ZY and HX Y. ZY, ZQ X and $H X Y$ were all responsible for the final submission. The author(s) read and approved the final manuscript.

\section{Authors' information}

Burn wounds are one field studied by $\mathrm{YZ}$ and $\mathrm{YHX}$, who are researchers at the First Affiliated Hospital of Nanchang University focusing on new treatments (especially emerging cell-based therapy) for such injuries.

\section{Funding}

This article was funded by the Key Research and Development of Jiangx Province (No. 20192BBGL70016).

\section{Availability of data and materials}

Not applicable.

\section{Ethics approval and consent to participate}

Not applicable.

\section{Consent for publication}

Not applicable.

\section{Competing interests}

There is no conflict of interests.

\section{Author details}

${ }^{1}$ The First Affiliated Hospital of Nanchang University, No. 17, Yongwai Zhengjie, Nanchang, JiangXi Province, China. ${ }^{2}$ Spine Surgery, Third Affiliated Hospital of Sun-Yat Sen University, No. 600, Tianhe Road, Tianhe District, Guangzhou, Guangdong Province, China.

Received: 14 May 2020 Revised: 23 June 2020

\section{Accepted: 6 August 2020 Published online: 28 August 2020}

\section{References}

1. World Health Organization. 2016 Burns Fact Sheet worldwide. 2017. http://www.who.int/mediacentre/factsheets/fs365/en/.

2. Nielson CB, Duethman NC, Howard JM, Moncure M, Wood JG. Burns: pathophysiology of systemic complications and current management. J Burn Care Res. 2017;38(1):e469-81.

3. Brosh K, Rozenman Y. Chemical burn-induced stromal demarcation line. Cornea. 2016;35(2):286-8.
4. Mai Y, Nishie W, Sato K, Hotta M, Izumi K, Ito K. Bullous pemphigoid triggered by thermal burn under medication with a dipeptidyl peptidase-IV inhibitor: a case report and review of the literature. Front Immunol. 2018;9:542.

5. Martin NA, Falder S. A review of the evidence for threshold of burn injury. Burns. 2017:43(8):1624-39.

6. Bittner EA, Shank E, Woodson L, Martyn JA. Acute and perioperative care of the burn-injured patient. Anesthesiology. 2015;122(2):448-64.

7. Peck MD. Epidemiology of burns throughout the world. Part I: Distribution and risk factors. Burns. 2011;37(7):1087-100.

8. Alessio V, Culler CA. Systemic and local management of burn wounds. Vet Clin North Am Small Anim Pract. 2017;6(47)

9. Vigani Alessio CCAS. Surgical treatment and management of the severely burn patient: review and update. Vet Clin North Am Small Anim Pract. 2017;6(47).

10. Mahmood R, Mehmood A, Choudhery MS, Awan SJ, Khan SN, Riazuddin S. Human neonatal stem cell-derived skin substitute improves healing of severe burn wounds in a rat model. Cell Biol Int. 2019;43(2):147-57.

11. Finnerty CC, Jeschke MG, Branski LK, Barret JP, Dziewulski P, Herndon DN. Hypertrophic scarring: the greatest unmet challenge after burn injury. Lancet. 2016;388(10052):1427-36.

12. Ghieh F, Jurjus R, Ibrahim A, et al. The use of stem cells in burn wound healing: a review. Biomed Res Int. 2015;2015:684084.

13. Hu MS, Borrelli MR, Lorenz HP, Longaker MT, Wan DC. Mesenchymal stromal cells and cutaneous wound healing: a comprehensive review of the background, role, and therapeutic potential. Stem Cells Int. 2018;2018: 6901983.

14. Lasocka I, Jastrzębska E, Szulc-Dąbrowska L, Skibniewski M, Pasternak I, Hubalek Kalbacova M. The effects of graphene and mesenchymal stem cells in cutaneous wound healing and their putative action mechanism. Int J Nanomedicine. 2019;14:2281-99.

15. Maranda EL, Rodriguez-Menocal L, Badiavas EV. Role of mesenchymal stem cells in dermal repair in burns and diabetic wounds. Curr Stem Cell Res Ther. 2017:1(12)

16. Shi X, Chen Q, Wang F. Mesenchymal stem cells for the treatment of ulcerative colitis: a systematic review and meta-analysis of experimental and clinical studies. Stem Cell Res Ther. 2019;10(1):212-66.

17. Curtis E, Martin JR, Gabel B, Sidhu N, Rzesiewicz TK, Mandeville R, Van Gorp S, Leerink M, Tadokoro T, Marsala S, Jamieson C. A first-in-human, phase I study of neural stem cell transplantation for chronic spinal cord injury. Cell Stem Cell. 2018;6(22):941-50.

18. Ma K, Tan Z, Zhang C, Fu X. Mesenchymal stem cells for sweat gland regeneration after burns: from possibility to reality. Burns. 2016;3(42):4617-25

19. Kanji S, Das H. Advances of stem cell therapeutics in cutaneous wound healing and regeneration. Mediators Inflamm. 2017;2017:5217967.

20. Jeschke MG, Rehou S, Mccann MR, Shahrokhi S. Allogeneic mesenchymal stem cells for treatment of severe burn injury. Stem Cell Res Ther. 2019; 10(1):336-7.

21. Mansilla E, Marin GH, Berges M, Scafatti S, Rivas J, Nunez A. Cadaveric bone marrow mesenchymal stem cells: first experience treating a patient with large severe burns. Burns Trauma. 2015;3:17.

22. Rasulov MF, Vasil'Chenkov AV, Onishchenko NA, Krasheninnikov ME, Kravchenko VI, Gorshenin TL, Pidtsan RE, Potapov IV. First experience in the use of bone marrow mesenchymal stem cells for the treatment of a patient with deep skin burns. Cell Technol Biol Med. 2005;1(6):141-4.

23. Abo-Elkheir W, Hamza F, Elmofty AM, Emam A, Abdl-Moktader M, Elsherefy $\mathrm{S}$, Gabr H. Role of cord blood and bone marrow mesenchymal stem cells in recent deep burn: a case-control prospective study. Am J Stem Cells. 2017; 3(6):23-5.

24. Liberati A, Altman DG, Tetzlaff J, Mulrow C, Gøtzsche PC, loannidis JPA. The PRISMA statement for reporting systematic reviews and meta-analyses of studies that evaluate health care interventions: explanation and elaboration. J Clin Epidemiol. 2009;62(10):e1-34.

25. Kaita Y, Tarui T, Yoshino H, Matsuda T, Yamaguchi Y, Nakagawa T. Sufficient therapeutic effect of cryopreserved frozen adipose-derived regenerative cells on burn wounds. Regen Ther. 2019;10(10):92-103.

26. Shumakov VI, Onishchenko NA, Rasulov MF, Krasheninnikov ME, Zaidenov VA. Mesenchymal bone marrow stem cells more effectively stimulate regeneration of deep burn wounds than embryonic fibroblasts. Bull Exp Biol Med. 2003:136(2):192-5.

27. Zhang J, La X, Fan L, et al. Original article immunosuppressive effects of mesenchymal stem cell transplantation in rat burn models. Int J Clin Exp Pathol. 2015;5(8):5129-36. 
28. Foubert P, Liu M, Anderson S, Rajoria R, Gutierrez D, Zafra D. Preclinical assessment of safety and efficacy of intravenous delivery of autologous adipose-derived regenerative cells (ADRCs) in the treatment of severe thermal burns using a porcine model. Burns. 2018;44(6):1531-42.

29. Peck MD, Kruger GE, van der Merwe AE, Godakumbura W, Ahuja RB. Burns and fires from non-electric domestic appliances in low and middle income countries. Burns. 2008;34(3):303-11.

30. Smolle C, Cambiaso-Daniel J, Forbes AA, Wurzer P, Hundeshagen G, Branski LK. Recent trends in burn epidemiology worldwide: a systematic review. Burns. 2017;43(2):249-57.

31. WHO. The injury chartbook: a graphical overview of the global burden of injuries. Geneva: World Health Organization; 2002. http://whqlibdoc.who.int/ publications/924156220X.pdf.

32. Friedenstein AJ, Chailakhjan RK, Lalykina KS. The development of fibroblast colonies in monolayer cultures of guinea-pig bone marrow and spleen cells. Cell Tissue Kinet. 1970;3(4):393-403.

33. Chamberlain G, Fox J, Ashton B, Middleton J. Concise review: mesenchymal stem cells: their phenotype, differentiation capacity, immunological features, and potential for homing. Stem Cells. 2007;25(11):2739-49.

34. Wexler SA, Donaldson C, Denning-Kendall P, Rice C, Bradley B, Hows JM. Adult bone marrow is a rich source of human mesenchymal 'stem' cells but umbilical cord and mobilized adult blood are not. Br J Haematol. 2003; 121(2):368-74

35. Salingcarnboriboon R, Yoshitake H, Tsuji K, et al. Establishment of tendonderived cell lines exhibiting pluripotent mesenchymal stem cell-like property. Exp Cell Res. 2003;2003(287):289-300.

36. Zuk PA, Zhu M, Mizuno H, Huang J, Futrell JW, Katz AJ. Multilineage cells from human adipose tissue: implications for cell-based therapies. Tissue Eng. 2001;7(2):211-28.

37. De Bari C, Dell'Accio F, Tylzanowski P. Multipotent mesenchymal stem cells from adult human synovial membrane. Arthritis Rheum. 2001;8(44):1928-42.

38. Toma JG, Akhavan M, Fernandes KJ, Barnabe-Heider F, Sadikot A, Kaplan DR. Isolation of multipotent adult stem cells from the dermis of mammalian skin. Nat Cell Biol. 2001;3(9):778-84

39. Seo B, Miura M, Gronthos S, Mark Bartold P, Batouli S, Brahim J. Investigation of multipotent postnatal stem cells from human periodontal ligament Lancet. 2004;364(9429):149-55.

40. Sotiropoulou PA, Perez SA, Gritzapis AD, Baxevanis CN, Papamichail M. Interactions Between Human Mesenchymal Stem Cells and Natural Killer Cells. Stem Cells. 2006;24(1):74-85.

41. Jiang X, Zhang Y, Liu B, Zhang S, Wu Y, Yu X. Human mesenchymal stem cells inhibit differentiation and function of monocyte-derived dendritic cells. Blood. 2005;105(10):4120-6.

42. Wu Y, Huang S, Enhe J, Ma K, Yang S, Sun T. Bone marrow-derived mesenchymal stem cell attenuates skin fibrosis development in mice. Int Wound J. 2014;11(6):701-10.

43. Maranda EL, Rodriguez-Menocal L, Badiavas EV. Role of mesenchymal stem cells in dermal repair in burns and diabetic wounds. Curr Stem Cell Res Ther. 2017:12(1):61-70.

44. Drago H, Marín GH, Sturla F, Roque G, Mártire K, Díaz Aquino V. The next generation of burns treatment: intelligent films and matrix, controlled enzymatic debridement, and adult stem cells. Transplant Proc. 2010;42(1): 345-9.

45. Burd A, Ahmed K, Lam S, Ayyappan T, Huang L. Stem cell strategies in burns care. Burns. 2007:33(3):282-91.

46. Natesan S, Wrice NL, Baer DG, Christy RJ. Debrided skin as a source of autologous stem cells for wound repair. Stem Cells. 2011;29(8):1219-30.

47. Branski LK, Gauglitz GG, Herndon DN, Jeschke MG. A review of gene and stem cell therapy in cutaneous wound healing. Burns. 2009:35(2):171-80.

48. Abbas OL, Özatik O, Gönen ZB, Öğüt S, Entok E, Özatik FY. Prevention of burn wound progression by mesenchymal stem cell transplantation. Ann Plast Surg. 2018;81(6):715-24

49. Francis $E$, Kearney $L$, Clover J. The effects of stem cells on burn wounds: a review. Int J Burns Trauma. 2019;9(1):1

50. Motamed S, Taghiabadi E, Molaei H, Niloofar S, Seyed Esmaeil H, Saeed S. et al. Cell-based skin substitutes accelerate regeneration of extensive burn wounds in rats. Am J Surg. 2017;214(4):762-9.

51. Sultan SM, Barr JS, Butala P, Davidson EH, Weinstein AL, Knobel D. Fat grafting accelerates revascularisation and decreases fibrosis following thermal injury. J Plast Reconstr Aesthet Surg. 2012;65(2):219-27.
52. Shi $Y, T u L X$, Deng $Q$, et al. Effects and mechanism of rat epidermal stem cells treated with exogenous vascular endothelial growth factor on healing of deep partial-thickness burn wounds in rats. Zhonghua Shao Shang Za Zhi. 2020;3(36):195-203.

53. Wu R, Du D, Bo Y, Zhang M, Zhang L, Yan Y. Hsp90a promotes the migration of iPSCs-derived keratinocyte to accelerate deep second-degree burn wound healing in mice. Biochem Biophys Res Commun. 2019;520(1): 145-51.

54. Kanji S, Das M, Aggarwal R, Lu J, Joseph M, Basu S. Nanofiber-expanded human umbilical cord blood-derived CD34+ cell therapy accelerates murine cutaneous wound closure by attenuating pro-inflammatory factors and secreting IL-10. Stem Cell Res. 2014;12(1):275-88.

55. Cardinal M, Eisenbud DE, Phillips T, Harding K. Early healing rates and wound area measurements are reliable predictors of later complete wound closure. Wound Repair Regen. 2008;16(1):19-22.

56. Clinicaltrials.gov, Stem Cell Therapy to Improve Burn Wound Healing. https://clinicaltrials.gov/ct2/show/NCT02104713\#wrapper. [52020]. Accessed 30 May 2020

57. Gentile P, Scioli MG, Cervelli V, Orlandi A, Garcovich S. Autologous micrografts from scalp tissue: trichoscopic and long-term clinical evaluation in male and female androgenetic alopecia. Biomed Res Int. 2020;2020: 7397162.

58. Gentile P. Autologous cellular method using micrografts of human adipose tissue derived follicle stem cells in androgenic alopecia. Int J Mol Sci. 2019; 20(14):3446

59. Cervelli V, Lucarini L, Spallone D, et al. Use of platelet-rich plasma and hyaluronic acid in the loss of substance with bone exposure. Adv Wound Care. 2011;24:176-81.

60. Nicoli F, Balzani A, Lazzeri D, et al. Severe hidradenitis suppurativa treatment using platelet-rich plasma gel and hyalomatrix. Int Wound J. 2013;12:338-43.

61. Cervelli V, Garcovich S, Bielli A, Cervelli G, Curcio BC, Scioli MG. The effect of autologous activated platelet rich plasma (AA-PRP) injection on pattern hair loss: clinical and histomorphometric evaluation. Biomed Res Int. 2014;2014: 760709.

62. Gentile P, Bottini DJ, Spallone D, Curcio BC, Cervelli V. Application of platelet-rich plasma in maxillofacial surgery: clinical evaluation. J Craniofac Surg. 2010;21(3):900-4

63. Scioli MG, Bielli A, Gentile P, Cervelli V, Orlandi A. Combined treatment with platelet-rich plasma and insulin favours chondrogenic and osteogenic differentiation of human adipose-derived stem cells in three-dimensional collagen scaffolds. Tissue Eng Regen Med. 2006;11(8):2398-2410.

64. Wang Y, Wang J, Li Y, Wang S, Zhu X. Platelet-rich Plasma Protects HUVECs against OX-LDL-induced Injury. Open Med (Wars). 2018;13:41-52.

65. Gentile P, Scioli GM, Bielli A, Orlandi A, Cervelli V. Comparing different nanofat procedures on scars: role of the stromal vascular fraction and its clinical implications. Regen Med. 2017:12(8):939-52.

66. Gentile P, Colicchia GM, Nicoli F, Cervelli G, Curcio CB, Brinci L. Complex abdominal wall repair using a porcine dermal matrix. Surg Innov. 2013;20(6): P12-5.

67. Gentile P, Calabrese C, De Angelis B, Pizzicannella J, Kothari A, Garcovich S. Impact of the different preparation methods to obtain human adiposederived stromal vascular fraction cells (AD-SVFs) and human adiposederived Mesenchymal stem cells (AD-MSCs): enzymatic digestion versus mechanical centrifugation. Int J Mol Sci. 2019;20(21):5471.

68. Gentile P, Garcovich S. Concise review: adipose-derived stem cells (ASCs) and adipocyte-secreted exosomal microRNA (A-SE-miR) modulate cancer growth and promote wound repair. J Clin Med. 2019;8(6):855.

\section{Publisher's Note}

Springer Nature remains neutral with regard to jurisdictional claims in published maps and institutional affiliations. 